\title{
State of the Art in Holographic Displays: A Survey
}

\author{
Fahri Yaraş, Graduate Student Member, IEEE, Hoonjong Kang, and Levent Onural, Fellow, IEEE
}

\begin{abstract}
True-3D imaging and display systems are based on physical duplication of light distribution. Holography is a true-3D technique. There are significant developments in electro-holographic displays in recent years. Liquid crystal, liquid crystal on silicon, optically addressed, mirror-based, holographic polymer-dispersed, and acousto-optic devices are used as holographic displays. There are complete electro-holographic display systems and some of them are already commercialized.
\end{abstract}

Index Terms-Holographic displays, digital holography, real-time holography, spatial light modulators (SLMs).

\section{INTRODUCTION}

I N THIS PAPER, we examined the current state of the art in holographic display technologies. 3D displays are expected to have a significant impact in various areas over the next decade. Although the basic stereoscopic 3D displays have been readily available for almost two decades, they failed to become a commercially successful consumer product. A reason for this is the viewing discomfort associated with stereoscopic approaches. Almost all currently available 3D displays are still stereoscopic. Although the advanced techniques, such as eyetracking, may reduce the discomfort to a certain degree, it is impossible to eliminate it totally due to the fundamental principles of stereoscopy. "True-3D" systems in which none of the restrictions on the viewer exists due to physical duplication of light distribution, is more desirable and superior compared to stereoscopy; however such display systems are much more complicated. Holography is a sophisticated true-3D method. More research is needed to overcome technological difficulties associated with holographic displays.

Holography is first presented in 1948 by D. Gabor (1900-1979) [1]-[3] to avoid aberrations in electron microscopy. However, since the quality of the images was not satisfactory, holography lost its popularity. After the developments in laser technologies, holography came forward again. Leith and Upatnieks [4]-[6] developed the off-axis holography and then Denisyuk invented the thick reflection holograms by combining the ideas of Gabor and Lippmann [7], [8]. Lohmann and Paris generated a hologram by using a digital computer in 1967 [9]; calculated holograms are printed on a transparency. Goodman and Lawrence proposed the idea of digital holography in 1967 [10] and in 1980 Yaroslavskii and Merzlyakov introduced the fundamental theory of digital holography.

Manuscript received December 01, 2009; revised March 03, 2010; accepted March 10, 2010. Date of publication May 27, 2010; date of current version August 27, 2010. This work is supported by EC within FP7 under Grant 216105 with the acronym Real 3D.

The authors are with the Bilkent University, Department of Electrical and Electronics Engineering, TR-06800 Ankara, Turkey (e-mail: fahri@ee.bilkent. edu.tr, hjkang@ee.bilkent.edu.tr, onural@ee.bilkent.edu.tr).

Color versions of one or more of the figures are available online at http:// ieeexplore.ieee.org.

Digital Object Identifier 10.1109/JDT.2010.2045734
There are some articles, surveys and reported workshops that are related to holography and its applications in the literature [11]-[17]. However over the years they became outdated and new technologies and methods emerged. Although we cited some of the old papers for the sake of completeness, the bulk of the paper is based primarily on recent publications. This paper will be useful to researchers in the field since it will provide a better understanding of the current state of the art; this will then guide subsequent research to a more meaningful direction. Secondly, the survey will contribute to the efficiency of research by preventing unnecessary duplication of already conducted research.

In this paper we first discussed technical issues. Spatial light modulators (SLMs) using liquid crystal, liquid crystal on silicon (LCoS), optically addressed, mirror-based, and holographic polymer-dispersed and acousto-optic devices are presented in Section II. We include recent publications on these topics and give detailed information on their operating principles and drawbacks. We report current electro-holographic display systems in Section III. Some of the holography related display methods, which can not be classified as purely holographic techniques, are included in Section III-H; these include holographic screens and some hybrid techniques. Finally, we present a critical analysis in the conclusion section.

\section{TECHNICAL ISSUES IN ELECTRO-HOLOGRAPHY}

\section{A. An Overview}

Conventional holographic films offer full-parallax and excellent resolution which is in the order of thousands of lines $/ \mathrm{mm}$. However such a resolution is not yet possible for electro-holographic recording. Another difficulty arises due to pixellated structures. Compared to wavelength of the visible monochromatic light, pixels are not small enough to yield sufficient viewing angles. Recent achievements in new generation of high efficiency liquid crystal devices have provided new tools for electro-holographic reconstructions from digital holograms.

Several approaches for electro-holographic reconstructions are reported. Holographic reconstruction by liquid crystal devices is one of the most common methods. Bauchert, Serati, and Furman [18] reported advances in liquid crystal SLMs. According to them, these improvements include higher pixel density, smaller pixel period, greatly improved optical efficiency, and higher speed operation. The progress in liquid crystal on silicon (LCoS) SLMs and their applications were presented by Michalkiewicz et al. [19]. In their work, they discussed several issues connected with electro-holographic reconstruction of digital holograms by means of LCoS devices. Moreover, various applications of digital holography that also include holographic reconstructions by SLMs were reviewed by Frauel et al. [20]. The presented applications cover three-dimensional (3D) imaging and the problems associated with it. Holographic reconstructions using reflective liquid 
crystal devices (LCDs) together with a light-emitting diode (LED) reference light are discussed in [21]. They presented the setup and results for electro-holographic reconstructions that are illuminated by a LED. For electro-holography, another approach is time and space multiplexing of SLMs. Shimobaba et al. [22], [23] analyzed an electro-holographic reconstruction method for a color 3D object, using time division switching of reference lights. A color 3D object is divided into red, green and blue components and they compute three computer-generated holograms (CGHs) from those components. A single LCD displays the CGHs in sequence at a total refresh rate of about $100 \mathrm{~Hz}$. In [24]-[26], Sato et al. proposed another time-multiplexed holographic color display system that uses a high-resolution reflective LCD which consists of a $1920 \times 1080$ array of square pixels with a pixel period of $8.1 \mu \mathrm{m}$. They used red, blue, and green lasers for illumination and high quality moving 3D color images are reconstructed by the developed holographic system. Moreover, the viewing zone is enlarged up to about 20 degrees by adopting a 6-channel LCD. Results of the color holographic system and possible further modifications are also discussed by the authors. The main differences between the system presented in [22], [23] and [24]-[26] are light sources and time-multiplexing methods. In [24]-[26] red, green, and blue lasers and an electronic shutter are used. On the other hand, in [22], [23] they use LEDs as light sources and replace the electronic shutter with directly switched LEDs. Another color holographic display system was proposed by Ito and Okano [27]. In this paper, the space-division method is used and three colored reference light beams illuminate a single SLM. In recent papers [28], [29], Ohmura and collaborators proposed a method to increase the viewing angle of the hologram by using a single spatial light modulator driven by a mirror module. With the help of the proposed method they doubled the resolution along the horizontal direction. As a result, the horizontal viewing angle was also doubled. In [30]-[33], Takano, Minami, and Sato developed a method for a color electro-holographic display system. A metal halide lamp and three SLMs were used and they made a comparison with systems that use lasers as light sources. In subsequent papers by Sato and Takano [34], [35] a full-color electro-holographic 3 -D display system employing LEDs is presented, and a virtual image reconstruction technique is studied. In addition, they proposed that direct and independent drives on LEDs through RGB waves bring ease to the adjustment of white balance, and a wide viewing angle can be attained. Another full-color holographic projection system by using the original parts of a projector is presented by Yamaguchi, Okabe and Yoshikawa [36], [37]. They used Fresnel holograms to speed up their system [37]. Reflective-type LCoS SLMs were also used by Moon and Kim in [38], [39]. Contrary to the three-SLM method, a full-color image pattern using a color wheel, by using only one $\mathrm{LCoS}$ SLM for full-color reconstruction of a 3D object is presented. The LCoS technology and LED illumination are used by Yaras et al. [40], [41]. In their work phase-only SLMs are used for reconstructions from inline phase holograms that are calculated by the Gerchberg-Saxton iterative algorithm. They have shown that reconstruction of $2 \mathrm{D}$ objects whose sizes are larger than SLM size is possible. Not only single plane 2D objects but also several objects in different depths are reconstructed using phase holograms by superposing their complex diffraction patterns. A group from Barcelona University combined two LCDs and they are used to display full complex Fresnel holograms [42]-[44]. One of them is working in real-only mode and the other is imaginary-only. In another paper, they used one of the modulators in amplitude-only mode and the other one in phase-only mode. They claim that it is possible to obtain full complex modulation by combining two configurations of the modulators, such that a larger part of the complex plane is densely covered.

There are some groups who are studying the phase-only holograms. The paper [45] gives an overview of using synthesized phase (SP) objects instead of amplitude-only or amplitude and phase objects. They used an iterative algorithm to calculate object-dependent phase distributions of SP-objects. They suggested to use this method for pattern recognition problems in hybrid optical-digital $4 \mathrm{~F}$ correlators. Although their scope is different then holographic displays, iterative algorithms for calculating synthesized-phase objects are interesting. They also published their experimental results in another paper [46]. Yet another paper from this group [47] describes the calculation of double-phase holograms (DPHs) by two different iterative methods. They reported that by using either one of the two methods, the reconstructions can reach up to 50 percent of the full size of a diffraction order of the reconstruction. They have also shown that reconstructed images can also be observed in $(+1 / 2$ or $-1 / 2)$ diffraction orders together with the $0,+1$, and -1 orders.

Phase holograms (kinoforms) have significant advantage over amplitude holograms when diffusely illuminated objects are used [48], [49]. The kinoform preserves only the phase information of the complex field. The main advantage of the kinoform is the high diffraction efficiency that theoretically can reach 100 percent. A high-resolution kinoform can be fabricated with lithography techniques. Real-time kinoform generation is reported in [50]. Another paper [51] focuses on kinoform synthesis using "two" object-dependent filters (amplitude and phase), that are on the object plane rather than one phase filter as usual, in the process of iteration. Two filters give two degrees of freedom and this accelerates the convergence and gives less amplitude variance in the reconstructed image. They have also stated that the described method is the most efficient one for binary objects and it is generalized for off-axis kinoforms. Another paper from the same group [52] focuses on similar problems as the previous one but they have used weighting coefficients in the amplitude filter and control the amplitude of the object in this case. Encoding many intensity distributions into a single phase-only hologram is the topic of the paper by Makowski et al. [53]. Fully customizable object planes can be located at different positions. In their experimental results they used eleven object planes and compared their results for two holographic methods: photographic and electron beam lithography. Moreover, the paper by Nakamura et al. [54] proposes a numerical method to get complex amplitude distribution of a 3D object from a digital hologram. The proposed method contains two processes: first they record the hologram of a 3D object by two image sensors; after this process they get the phase. The second process is used to determine the phase distribution by an iterative algorithm. There are two constraints used in the algorithm: reconstructed 3D object is in focus and its 


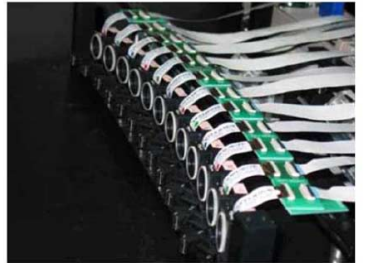

(a)

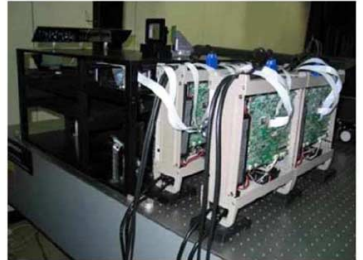

(b)
Fig. 1. Pictures of the dynamic holographic stereogram. (a) Curved array of SLMs mounted without upper arms. (b) Whole system with electronic controllers [57]. (J. Hahn, H. Kim, Y. Lim, G. Park, and B. Lee, "Wide viewing angle dynamic holographic stereogram with a curved array of spatial light modulators," Opt. Express, 16, 12372-12386 (2008),@ 2008 OSA. Reprinted with permission.)

conjugate is out of focus. They have also shown the numerical and experimental results of the proposed algorithm.

A zeroth-order suppression method is proposed by Palima and Daria [55]. They introduce corrections to the phase-only CGHs. Corrections are made by designing a hologram to produce a tailored light pattern and they used a corrective beam that destructively interferes with the zeroth-order beam. With the help of the proposed technique, removal of the zeroth-order beam due to partial reflection from the SLMs protective glass plates and coatings is also achieved. Yet another group from Korea, who are mostly specialized for integral imaging, proposed a novel full parallax viewing-angle enhanced computer generated holographic 3D display system [56]. They also implemented the proposed system by combining a micro-lens array and colorized synthetic phase holograms displayed on a phase-type spatial light modulator. In order to analyze the viewing-angle limitations of their 3D display system, they provide some theoretical background and introduce a simple ray-tracing method for 3D image reconstruction. With the help of proposed method they report continuously varying full parallax 3D images with a viewing angle of about $\pm 6^{\circ}$. Another novel design of holographic display is proposed by the same group, recently [57]. Their system is described as a kind of holographic stereogram with a curved array of SLMs (Fig. 1). Based on the fundamental idea of the holographic stereogram, each SLM in the system is individually transformed to display the local angular spectra of object wave in the curved array of SLMs. Each SLM is reconfigured so that the optical structure is simplified and the light power loss is reduced. They reported that this design improves the viewing angle of holographic display and the loss of light power is significantly reduced. With this method, they achieved a viewing angle of about $22.8^{\circ}$.

A SLM-based implementation of a method to create complex valued holograms by using two cascaded phase diffractive optical elements (DOEs) have been presented by Jesacher $\mathrm{et} \mathrm{al}$. [58]. By diffracting the light from two successive phase holograms that are located in conjugate Fourier planes, they can program both spatial amplitude and phase distributions independently. They also show that the obtained light efficiency is close to 100 percent.

For all these holographic applications, SLMs are among the most popular devices. SLM is a device that can modulate light in a prescribed way, based on its input control parameters. The two main types are optically and electrically addressed SLMs. With an optically addressed SLM a coherent light source is used to write the prescribed spatial pattern onto the SLM. The SLM is then used to modulate the light from a different monochromatic light source. Electrically addressed SLMs can convert electrical signals into an interference pattern. Typically, electrically addressed SLMs are popular since they are readily addressed from a computer. Existing types of SLMs include LCDs, acousto-optical displays and digital micro-mirror devices (DMDs). The main requirements of an SLM to be used in digital holography are that they should be fast, have high transmittance and provide a good optical efficiency [59].

The wavefront of an optical field can be controlled by using liquid crystal devices [42]-[44], [60]-[67]. Moreover, this can be done in real time. To drive a liquid crystal display, we need two inputs. One of them is the applied voltage to the pixel. The other input is the polarization state of the incident light. Another factor that determines the wavefront obtained by using a liquid crystal display is its configuration. There are three common configurations used to display images on a LCD. First one is called the "high-contrast (HC)" method [42]. In this configuration the aim is to achieve highest contrast ratio, but there may be a significant phase modulation. The second one is called the "amplitude mostly" method [42]; it is used to modulate the amplitude, but its contrast ratio is lower than $\mathrm{HC}$ configuration and also there is still a small phase variation on the output wavefront. The third one is the so-called "phase-mostly" configuration [42]; it provides phase modulation but there is always a small variation in amplitude, as well. Several methods are proposed to modulate a wavefront by using the above mentioned LCD configurations. One of them is to codify the complex information to be displayed on a single device within its limited capabilities. Birch et al. used a 128 by 128 analog ferroelectric liquid crystal SLM and to achieve full complex modulation in the Fourier plane they used two pixels as a macro pixel [62]. One of the pixels is used for the real part and the other one is used for the imaginary part of the complex field. However, such an approach causes degradation in resolution. Moreover, there is an intensity modulation on the output field. This can be useful to eliminate the conjugate image (the twin image) which overlaps on the real image. Another example is proposed by Stolz et al. [63] and their coding algorithm has eight-levels of quantization. They used twisted nematic liquid crystal SLM to couple the phase and amplitude information. A twisted nematic liquid crystal SLM has a spiral response in the complex plane. They indicate that coupling between the amplitude and the phase brings a trade-off between the diffraction efficiency and the degradation on the quality of reconstructed object. According to their conclusion, the twisted nematic liquid crystal SLM can provide a phase modulation up to $\pi$ radians or more and can also offer a high transmittance. Tudela et al. also used two LCDs to have complex modulation but they proposed a simpler optical setup [42]. Their method is based on adaptation of the complex information to real devices. The first step is to adjust the amplitude information according to the response of the high-contrast LCD. The second step is to adjust the phase together with the compensation of the phase distortion introduced by the amplitude stage. Adjustment in step two is computed by using the minimum Euclidean distance criteria.

SLM properties and their settings were discussed by the group of Kohler et al. [68]. They concluded that the quality 
of the reconstructed holograms depends on the properties of the modulator. Therefore they stated that the characterization of the modulators is required. They showed the result of a modulator characterization and the influence of the modulator on the quality of the reconstructed holograms. However, due to the production process, the reflective surfaces are generally not flat; therefore this leads to unwanted distortions in the phase of the light. Due to this deviation, holographic reconstructions may be affected adversely. Jesacher et al. [69] also proposed a non-interferometric method based on the Gerchberg-Saxton algorithm to correct those surface aberrations. The information about the surface distortion is obtained from the shape of a single optical vortex which is created by the light modulator. Another development about SLMs has been presented by Putten [70]. In their paper full spatial phase and amplitude control of a laser beam using a twisted nematic LCD combined with a spatial filter is presented. Their modulation scheme combines four neighboring pixels into one super-pixel. Each super-pixel is able to independently generate any complex amplitude value. They showed that they are able to freely modulate the phase over a range of $2 \pi$ while keeping the amplitude of the light constant. The advantage of the presented method is that the modulation technique can be used with a wide range of different SLMs. Another calibration technique for SLMs to have better holographic reconstructions is reported by Otón et al. [71]. A multiple look-up table method provides multi-point calibration to compensate both for the backplane curvature and for other possible nonuniformities caused by thickness variations of the liquid crystal layer.

\section{B. Liquid Cristal Devices}

In 1888 Austrian botanist Friedrich Reinitzer discovered the liquid crystal [72]. A liquid crystal has a number of advantageous characteristics that make it very consistent for displays. Most of these characteristics arise due to the anisotropic nature of the material. This means that the liquid crystal can react differently to different forms of illumination. Moreover, their optical properties may be varied depending on the electric or magnetic fields applied to the liquid crystal. These characteristics can be used for designing displays. Liquid crystal SLMs are electrically addressed devices with a driving mechanism similar to that used in commercially available LCD TVs. Dynamic interference patterns can be written on liquid crystal (LC) SLMs. The two main types are twisted nematic and ferroelectric LC SLMs. LC SLMs can be used to perform amplitude and phase modulation on the illuminating source. Since either amplitude or phase modulation may be employed in holography, a choice must be made. Phase modulation is often chosen over amplitude modulation, since it does not require the additional polarization optics which reduce the optical efficiency of the device. A LC SLM consists of a matrix structure of twisted nematic cells that may be individually addressed. The liquid crystal molecules are sandwiched between two glass substrates and the orientation of the molecules gradually twisted to $90^{\circ}$ [73]. On reception of linearly polarized light, its polarization is twisted to $90^{\circ}$. The LC molecules have to be aligned with an electric field to prevent a shift in polarization of the light. The cross polarization or parallel polarization of the cell allows to control the electric filed though the cell. As a result, amplitude modulation is achieved. Liquid crystal cells may operate as phase modulators by utilizing the dielectric anisotropy of the LC molecules. A LC cell, whose molecules are not twisted, is used when modulating the phase.

Transmissive LCDs are designed to transmit the backlight, through the liquid crystal layer. For transmissive displays a thin film of liquid crystals is placed between two thin pieces of glass [74]. Depending on the orientation of the liquid crystals and incident light, the polarization and the phase of the light travelling through the liquid crystal film is modified by a refractive index change in the liquid crystal layer. An additional polarizer in front and behind the liquid crystal film can be used for intensity modification of the passing light wave, by polarization changes in the liquid crystal layer. These effects can be varied by the type of liquid crystal and by the film thickness. Each pixel consists of an active area and a passive area where, for example, a transistor for the pixel control is located. When reducing the pixel size, the transistor cannot be reduced in size as much as the active area of the pixel. Consequently, the fill-factor, which specifies the percentage of the active area of a pixel, decreases when the size of the pixel is reduced. The effect of this reduced fill-factor is a reduction of the transmitted light efficiency. To overcome this problem, new transmissive LCDs with small pixel size have a micro-lens in front of each pixel to focus the light onto the active area, so that the light can be transmitted with better efficiency.

\section{LCoS Devices}

Liquid crystal on silicon (LCoS) devices use a combination of a liquid crystal and a mirror to perform optical modulation. The back of the mirror is designed as an electrode to apply the electric field to the liquid crystal layer. As with the transmissive LCD, the polarization and the phase of the light is modified by the LC due to the refractive index change in the liquid crystal layer. The thickness of the liquid crystals can be changed to obtain the same phase shift or polarization change because the light is passing through the film twice. The main advantage of LCoS technology is the high fill factor of up to 93\% [75], [76]. Such a high fill factor is achievable because the rear of the mirror is used as an electrode. There is, therefore, no need for a transistor beside each pixel. Today, LCoS SLMs can support $1920 \times 1080$ pixels and have pixel size down to $8 \mu \mathrm{m}$ [76]. The LCoS displays have a much better optical efficiency and a smaller pixel size than the transmissive displays. Therefore the suitability for holographic applications is better. Most LCoS displays use a very small liquid crystal film to obtain short switching times. A small liquid crystal layer leads to a small phase lag of the wavefront passing through the layer.

\section{Optically Addressed LCDs (OALCDs)}

An optically addressed LCD (OALCD) converts an intensity pattern into a phase or amplitude modulation. The backside (writing side) of the display is illuminated by an intensity pattern which creates a corresponding 2D-modulation of the refractive index in the liquid crystal film on the other side of the display (reading side) [74]. The incoherent light is converted into a voltage applied to the liquid crystal layer. Therefore the refractive index change is dependent on the incident intensity. 
The big advantage of the optical addressable LCD is the absence of pixels. Therefore no higher diffraction order occurs and hence a higher efficiency is possible. A drawback of the display is the low spatial resolution ( $\approx 50$ lines $/ \mathrm{mm}$ ) compared to analog holography as a consequence of physical limitations which are mainly due to used photo-conductors [77].

\section{E. Mirror-Based Devices}

Besides the previously discussed liquid crystal based light modulators, digital mirror devices form another group of systems for spatial light modulation. These types of light modulators consist of an array of micro-mirrors, where each mirror can be separately electro-mechanically controlled [78]. Basically, there are currently two types of DMDs; one where the mirrors can only be tilted, and the other one, where the mirrors can perform an out-of-plane linear translation. While those in the first group are used to modulate the intensity of the light field, the latter ones modulate the phase. One advantage of DMDs over LCDs is that the incident light is reflected with high efficiency, while the liquid crystal systems always suffer from a certain amount of light absorption, even if they are changing only the phase. This cannot be avoided, since the light always has to pass through the liquid crystal device. Additionally, because of the lower absorption, the mirror based devices can be used with higher light intensities without running into thermal problems. There is much development going on in the area of DMDs.

\section{F. Holographic PDLCs}

Polymer-dispersed liquid crystals (PDLCs) and holographic polymer-dispersed liquid crystals (HPDLC) are two of the most investigated materials for display production. The difference in refractive index between droplets and their environment changes as a result of applied electric field to the PDLC. The direction of the droplets align with the applied field and thus scattering characteristics of the PDLC changes. This in turn controls the light passes through. When there is no applied voltage on PDLC, orientation of the droplets become random and the PDLC behaves as a scattering object. These droplets are fabricated by dispersing LC nano-scale droplets in photo-polymer films. Main advantages are low cost, high resolution, high contrast, full color support, low power consumption, and low switch-on/switch-off durations. The major disadvantages are high control voltage (more than $100 \mathrm{~V}$ ) and limited viewing angle.

R. Pogue et al. in [79] made an extensive review of the PDLCs. An example of a 3D display constructed by the combination of PDLC and integral photography is discussed by Park et al. [80]. Another group from CLOSPI-BAS has been investigating the related problems and their solutions [81]-[83]. They studied holographic gratings to record them in polymer-dispersed liquid crystals. They also proposed methods to decrease the switching voltage.

\section{G. Acousto-Optical Modulators (AOMs)}

Acousto-optic (AO) SLMs have been used in real time 3D holographic video since 1989 at the MIT Media Lab [84], [85]. S. A. Benton first succeeded in producing computer generated electro-holograms using an $\mathrm{AO}$ device. AO devices are acoustically addressed light modulators that utilize the interaction of traveling acoustic waves and a coherent light source within a medium, to modulate the properties of the transmitted optical wavefront. The acousto-optic medium may consist of a piezo-electric transducer bonded to a suitable crystal such as fused silica. Upon application of an RF signal to the medium, the acoustic wave acts like a "phase grating" traveling through the crystal at the acoustic velocity of the material and with an acoustic wavelength dependent on the frequency of the RF signal. The incident laser beam is then diffracted by this grating. AO SLMs generate a 1D modulation and require a scanning mechanism. The scanning optics in an acousto-optical modulator $(\mathrm{AOM})$ system require synchronization to the fringe data stream and an optical processing unit for the reconstruction.

Onural et al. have proposed an acousto-optical holographic display device [86]. By using travelling surface-acoustic waves (SAWs), hologram is produced as a surface pattern. When electrical signals applied to the electrodes of the SAW device, a time-varying acoustical wave is generated on the surface of the crystal. Time-varying SAW pattern is controlled by signals that are applied to each electrode, simultaneously.

\section{ElECTRO-HOLOGRAPHIC DisPlay SySTEMS}

\section{A. Holo-Video}

The first practical electro-holographic display named Mark-I was developed by the Spatial Imaging Group at MIT Media Lab in 1989 [85], [87]-[93]. The Mark-I is capable of rendering full color $25 \mathrm{~mm} \times 25 \mathrm{~mm} \times 25 \mathrm{~mm}$ images with 15 degrees of viewing angle at rates around 20 frames per second (fps). After that, the second prototype display named Mark-II was demonstrated in 1992. The Mark-II provides $150 \mathrm{~mm} \times 75 \mathrm{~mm} \times 150 \mathrm{~mm}$ images with 36 degrees of viewing angle at rates of around 2.5 fps. Moreover, a next generation of holographic video display is developed for commercial purposes [94].

The early Mark-I electro-holography system provided either monochromatic or full-color display of holographic images by trading off vertical spatial resolution for three separate color channels. The holographic image is generated using a threechannel tellurium-dioxide $\left(\mathrm{TeO}_{2}\right)$ AOM. A holographic fringe pattern is sent through each channel of the AOM to modulate an RGB light consisting of three separate lasers. Each horizontal line has $32 \mathrm{~K}$ samples per color, and this provides sufficient resolution for the holographic diffraction pattern [84].

The second generation MIT holo-video display, or Mark II, used 18-channel AOM instead of a three-channel AOM and a bank of scanning mirrors instead of a rotating polygon. This version of the display could be scaled by simply adding more AOM channels and more scanning mirrors. The Mark II was a sixfold scale up from the previous display with an image volume of $150 \mathrm{~mm} \times 75 \mathrm{~mm} \times 150 \mathrm{~mm}(W \times H \times D)$ and 30 degrees of viewing angle. The Mark II was driven by a custom-built supercomputer called Cheops designed and implemented at the MIT Media Lab [87].

In Mark III, the AOM in which acoustic waves travel through the volume of a crystal, was replaced by a SAW device called a guided-wave optical scanner that uses acoustic waves traveling along the surface of the crystal [94]. Lithium niobate, which has a much lower acoustic attenuation than $\mathrm{TeO}_{2}$ and is useful for 


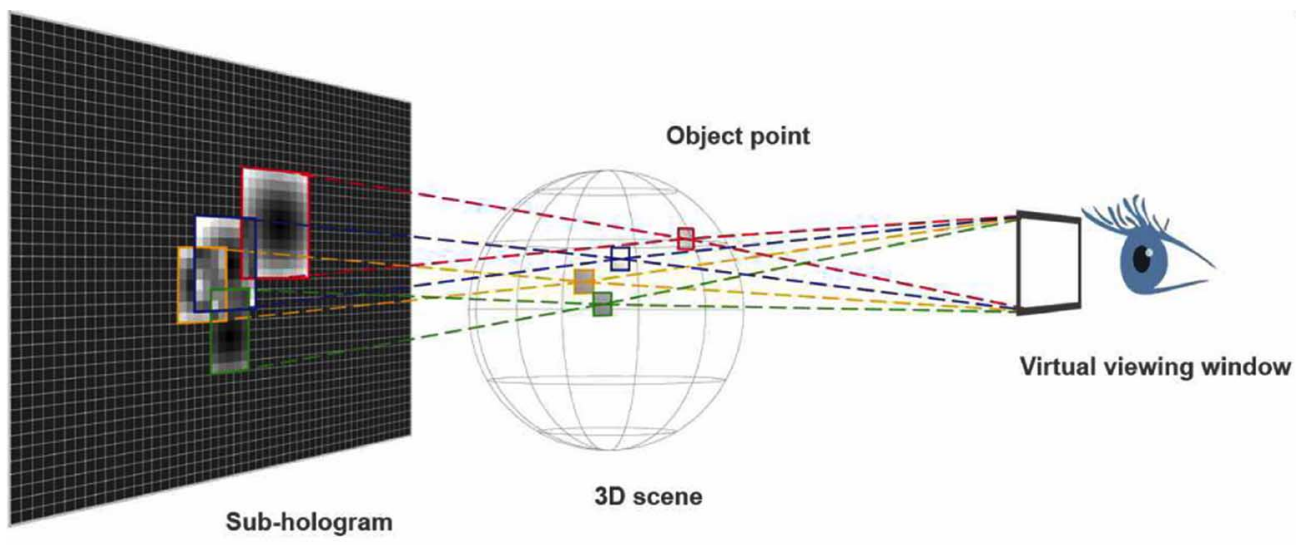

Hologram display

Fig. 2. Holographic display with a reconstructed 3D scene that is composed of object points. Each object point is encoded in a sub-hologram. The position and the size of a sub-hologram is determined by the object point position and the position of the virtual viewing window. The total hologram is generated by a summation of the sub-holograms. The 3D scene is visible through the virtual viewing window which is tracked to the eye position. The virtual viewing window contains the wavefront that would be generated by a real 3D scene at the eye position. (@ 2009 SeeReal. Reprinted with permission.)

modulating light with acoustic frequencies well into the gigahertz $(\mathrm{GHz})$ range, is used for these devices. The horizontal array of scanning mirrors is replaced in this generation by a holographic optical element (HOE). Mark-III has 440 scan lines and operates at $30 \mathrm{~Hz}$. The viewing angle is 24 degrees and the viewing volume is $80 \mathrm{~mm} \times 60 \mathrm{~mm} \times 80 \mathrm{~mm}(W \times H \times D)$.

\section{B. SeeReal}

SeeReal Technologies has developed a new approach to holographic displays. The primary goal of this approach is to reconstruct only that part of the wavefront originating from the object that actually hits the eye pupils of an observer [95]-[99]. It is a vertical-parallax-only system. A wavefront in a small region, called "observer window" is reconstructed. The size of the observer window is related to the size of the eye pupil. The observer window may be located in the Fourier plane of the hologram. A separate observer window for each eye is generated either by spatial or by temporal multiplexing. Then the size of the observer window determines the pixel period needed on the display. Closely related to the concept of the observer window is the subhologram which is a limited hologram region related to the positions of an object point and the eye pupil. Fig. 2 shows object points located at different depths and their subholograms with different sizes.

A large reconstruction volume of the 3D scene is possible. The size of the reconstruction is only limited by the size of the hologram display used. The reconstruction is located in a frustum ranging from the observer window to the borders of the display and beyond. Movement of the observer is allowed and tracked. The observer window can be shifted to the actual eye position by movement of the light source. A scene reconstruction is visible for an eye located inside the observer window. In Fig. 2, this reconstruction is shown between the display and the user; in general it can also be located fully or partly behind the display.

A monochrome real-time prototype using a 20-inch monitor has been built and demonstrated in 2007 (Fig. 3). A holographic reconstruction of the wave field that would be generated by a real object is obtained at the observer's eye position, and nowhere else. This special configuration allows to use displays with a relatively large pixel size. In the 20 -inch prototype, a state-of-the-art high resolution desktop LCD panel was used with separation of about $70 \mu \mathrm{m} \times 210 \mu \mathrm{m}$ between adjacent pixels.

Since the pixel size of the used SLM (the LCD panel) is large, the diffraction angle is about 0.5 degree, and the viewing window is also very small. However, a clear large reconstruction can be displayed on the space with a wide depth range of about 4 meters, because of the large SLM with a light steering technique (movement of the light source). An eye tracking system using two CCD cameras is also integrated to this prototype. According to the tracked eye position, the light path is adjusted to match the viewing window to the eye position in real time. The correct hologram which will generate the correct image and parallax from the observed position is computed and written to the SLM in real-time.

\section{QinetiQ}

The so-called Active Tiling system [100] was developed by QinetiQ in 2003. This system take advantage of the high frame rate of electrically addressed SLMs (EASLMs) and non-pixellated structure of optically addressed SLMs (OASLMs) [101]. It results in a system that can display images or patterns with significantly higher pixel counts than previously reachable. This design enables higher pixel counts to be written while maintaining the overall video update rates. The modulator system is composed of an EASLM to act as an "image engine" that can display the CGH image elements quickly; a replication optics set to project multiple de-magnified images of the EASLM onto an OASLM; OASLMs to store and display the computer-generated pattern; a readout optics set to form the holographic image; and a control system to synchronize the complete system (Fig. 4). This modulator system is designed to allow multiple channels to be assembled to produce a continuous output modulation plane and, system performance can be adapted or extended to meet different application requirements. OASLMs use optical 


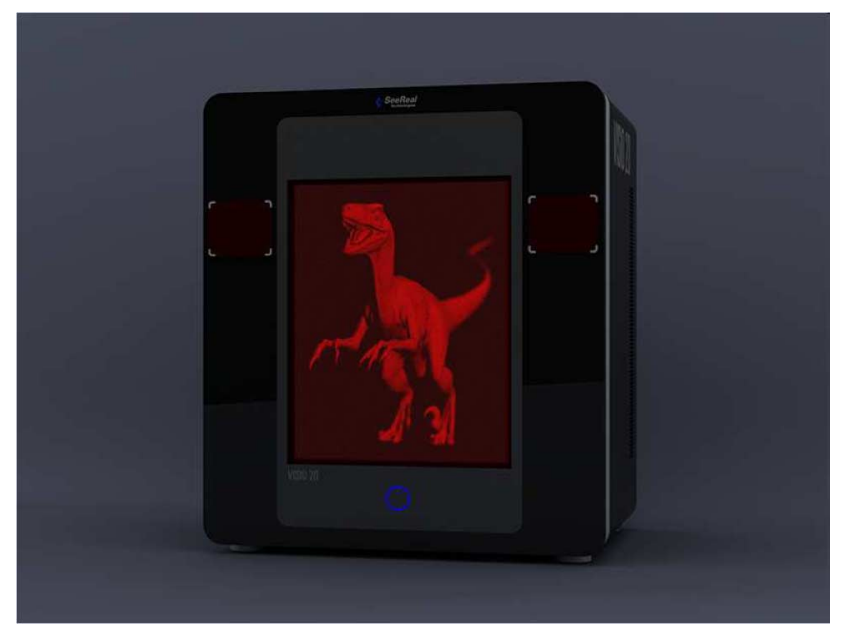

Fig. 3. Illustration of SeeReal's holographic display prototype with a screen diagonal of 20 inch. (@ 2009 SeeReal. Reprinted with permission.)

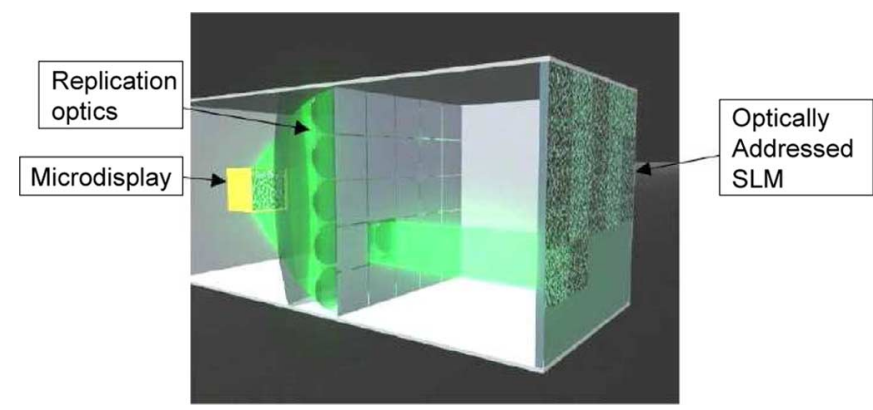

Fig. 4. Illustration of one channel of the Active Tiling modulator concept of QinetiQ [102] (Slinger, C., Cameron, C., Stanley, M., "Computer-Generated Holography as a Generic Display Technology," Computer, vol.38, no.8, pp. 46-53, Aug. 2005. (C) 2009 IEEE. Reprinted with permission.)

intensity patterns to modulate light, whereas EASLMs use electrical signals from electrical conductors.

A typical Active Tiling channel configuration consists of a ferroelectric crystal on silicon (FLCoS) EASLM with $1024 \times 1024$ binary pixels and operating at a $2.5-\mathrm{kHz}$ frame rate, a binary-phase diffractive optical element and associated refractive optics to perform the $5 \times 5$ replication, an OASLM, light-blocking layers, a dielectric mirror, and a ferroelectric liquid crystal output layer [102]. The output for each channel consists of 26 million pixels. Current Active Tiling system has more than $2.2 \times 10^{6}$ pixels $\mathrm{cm}^{-2}$ pixel area density. This density for a compact system volume exceeds $2.4 \times 10^{9}$ pixels $\mathrm{m}^{-3}$. Binary pixels in the system have a spacing of $6.6 \mu \mathrm{m}$ between them. The system has an updateable $1 \times 4$ channel arrangement $(5120 \times 20480$ pixels $=104$ megapixels $)$ in both monochromatic and frame-sequential-color operation. With the help of the scalable tilings, the system can have a pixel count in the order of $10^{9}$. Fig. 5 shows a reconstructed full-color 3D image with full-parallax by using spatially multiplexed $3 \times 8$ billion pixels.

\section{Horn}

The dedicated CGH calculators, named Horn (HOlographic ReconstructioN), by using field programmable gate array (FPGA) have being developed by the Tomoyoshi Ito's Lab

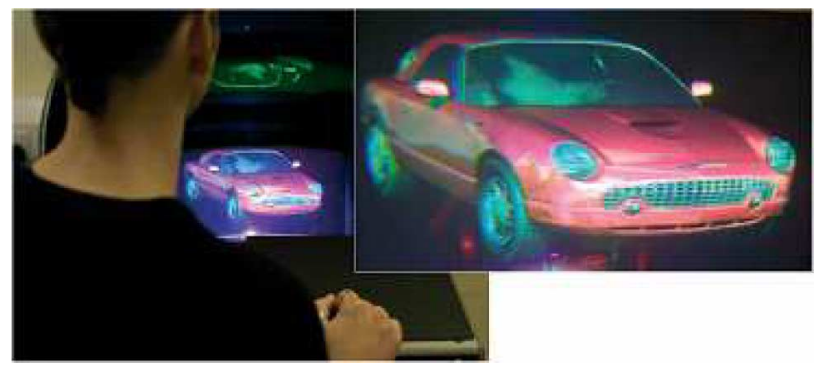

Fig. 5. Replay of a spatial-multiplexed, $3 \times 8$ billion-pixel, full-parallax, fullcolor, 3D image [102]. (Slinger, C., Cameron, C., Stanley, M., "Computer-Generated Holography as a Generic Display Technology," Computer, vol.38, no.8, pp. 46-53, Aug. 2005. ( 2009 IEEE. Reprinted with permission.)

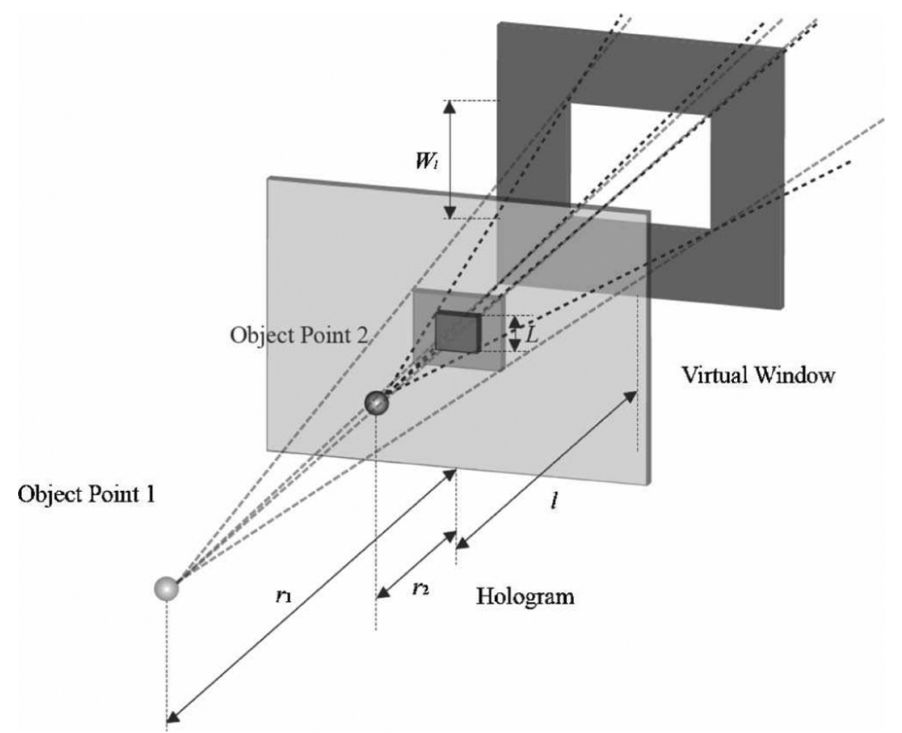

Fig. 6. Virtual window for image hologram calculation [111]. ("Real-time image plane full-color and full-parallax holographic video display system," T. Yamaguchi, G. Okabe, and H. Yoshikawa; Opt. Eng. 46, 125801 (2007). (C) 2009 SPIE. Reprinted with permission.)

research group in Chiba University since 1992 [103]-[109]. The first calculator, HORN-1, was developed in 1993, and its computing performance was about 300 megaflops. The HORN-6, latest version, was developed in 2008, and has four FPGA chips for calculation. The computing performance of the HORN-6 was improved compared to the HORN-5 through double data rate-synchronous dynamic random access memory (DDR-SDRAM) module integration to the board. Moreover, in order to achieve high performance and high quality, the approximated Fresnel hologram generation algorithm with a look-up table was used in the kernel of the HORN-6. Therefore, fringe patterns for 10000 object points can be calculated in real-time. Frame rates depend on the number of object points: 78 fps can be achieved for 1000 object points, whereas the rate drops to about $1 \mathrm{fps}$ for 200000 object points.

\section{E. Image Hologram}

Image hologram is the name given to the technique to record a real image of the object instead of the object itself by using a large lens [110]-[113]. The image of the object is located very close to the hologram; therefore, a single object image point contributes only to a small portion of the hologram. Therefore, 


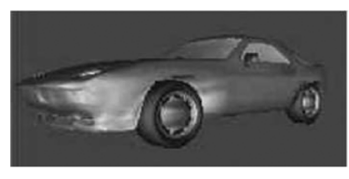

(a)

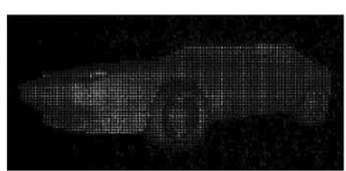

(b)

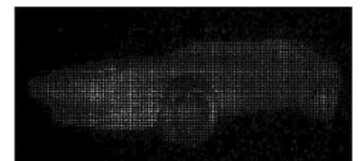

(c)

Fig. 7. A perspective image of the 3D model and reconstructed images (a) 3D Model (b) Direct reconstruction (c) ACPAS reconstruction [120].

computations per point are limited to a small portion. The advantage of such an approach is faster computation.

Fig. 6 shows the optical schematic which indicates the object point, corresponding fringe area for calculations, and the viewing zone (virtual window). The calculation time could be reduced by decreasing the distance between the object image point and the hologram plane, and increasing the distance between the hologram plane and the virtual window. The developed system in Nihon University has $1408 \times 1058$ pixel SLMs with a separation between centers of adjacent pixels as $10.4 \mu \mathrm{m}$. Number of object points is 3000 and a typical computing time is $60 \mathrm{~ms}$. They use white LED as the light source.

\section{F. Coherent Stereogram}

Computational speed of the coherent holographic stereogram is much faster than that of the conventional techniques [114]. The main reason for the speed up is the initial partitioning of the output fringe pattern into segments, and then approximating the contribution of each object point to the hologram pattern over one segment as a single 2D complex sinusoid; an initial Fourier domain pattern is composed by writing the complex amplitude of each such complex sinusoid to their frequency locations, and a subsequent inverse fast Fourier transform (IFFT) completes the fringe pattern computation for a segment. Repeating this procedure for each segment completes the computation. Modified and improved versions of the basic coherent stereogram are reported in the literature [115]-[120].

The latest version of the coherent stereogram is the accurate compensated phase-added stereogram (ACPAS), and yields a higher quality reconstructed image. The ACPAS is generated using both the fringe generation methods of the compensated phase-added stereogram and the accurate phase-added stereogram. This method generates more accurate results which closely resembles the results from conventional algorithms as shown in Fig. 7.

Since the algorithm of the coherent stereogram can be easily mapped to a single instruction multiple data architecture, the ACPAS algorithm can significantly benefit from graphics processing unit (GPU) based implementations. By using this method, one megapixel frame size full color holograms can be generated as a rate of $30 \mathrm{fps}$ for objects with more than 10000 points [121]-[126].

\section{G. NICT Holography System}

An electronic holography system was demonstrated at National Association of Broadcasters (NAB) exhibit in 2009 by Japan's National Institute of Information and Communications Technologies (NICT) [127]. The demonstrated system consists of three parts: a real scene acquisition system, a computing system, and an electro-holographic display system. The acquisition system uses an integral photography (IP) camera. It is an ultra-high-definition camera $(8 \mathrm{~K} \times 4 \mathrm{~K})$ shooting a real scene through a micro-lens array. A computer processes the image to generate a digital hologram from the captured image. The generated digital hologram is displayed on three LCDs $(4 \mathrm{~K} \times 2 \mathrm{~K})$ which are used as SLMs. Then, the SLMs are illuminated by the three primary color lasers (red, green, and blue) in order to reconstruct the elemental images from the holograms in space. All steps are processed in real-time. This prototype uses micro LCD SLMs, so the reconstruction size is small, but the impressive part is the ability to combine a live integral photography capture system with real-time holographic calculations and a subsequent holographic display.

\section{H. Other Approaches}

This section is devoted to approaches that can not be easily categorized into one of the previously discussed methods.

An interesting approach is described in a patent by Grossetie [128]. In this approach, the digital representation of the 3D object is sliced into $2 \mathrm{D}$ profiles and the complex wave field resulting from each is computed and then reconstructed. Timemultiplexed reconstruction of the profiles results in a human visual perception of the $3 \mathrm{D}$ object.

Holografika provides another type of 3D display system [129], [130]. Holografika products, such as HoloVizio, are essentially not holographic systems. They use only some holographic diffusers. The pixels of the holographic screen emit light beams to the various directions. These directed beams have different intensity and color values. The light beams are generated in the optical modules and then they hit the screen in various angles. Then the holographic screen makes an optical transformation to generate a continuous 3D view with vertical parallax [131]. Light beams which are leaving the pixels propagate in multiple directions through a software control, as if they were emitted from the points of 3D objects at fixed spatial locations. They have presented 50 megapixel, 10 megapixel, and 7.4 megapixel systems.

\section{Summary AND CRITICAL ANALYSIS}

Re-writeable holographic display devices primarily by electronic means are the main focus of this paper. Although advanced techniques such as auto-stereoscopy may relieve some of the inconveniences of stereoscopic systems to some extent, there are still fundamental problems associated with stereoscopy. Therefore the need for "True-3D" for a superior 3D displays is clear. True-3D can be explained as physical duplication of light distribution in a volume of interest. True-3D display systems are desirable and superior since none of the restrictions associated with stereoscopy exists in such displays. However true-3D displays are much more complicated. One of the sophisticated methods from true-3D display is holography. 
LCDs are one of the common choices as electro-holographic displays. Transmissive and reflective types are discussed in the paper. In transmissive type SLMs there are usually unwanted higher order diffraction based replications in the reconstruction. In addition to that, there might be also undiffracted light which degrades the reconstructions. We have seen that diffraction efficiency of commonly available reflective type SLMs is much higher. Therefore, such devices may be used to reduce the unwanted higher order diffractions and undiffracted beam. It is given in the literature that experiments with LCoS SLMs are promising. Another type of SLMs is optically addressed SLMs. These devices are rarely used in electro-holography. It seems that they have advantageous properties since they do not have a pixelated structure and the viewing angle is limited only by the wavelength of light. However their spatial resolution is low due to physical limitations of photo-conductors. Mirror-based SLMs are also used for holographic display systems. They are relatively fast devices and suitable for future electro-holography applications. However as all other devices, mirror-based SLMs also have some drawbacks: mostly they are binary modulators and generate gray levels only by time alternating methods. Another SLM type discussed in this survey is holographic polymer dispersed liquid crystals. Orientation of the nano-scale LC droplets in photo-polymer films can be controlled by the applied voltage in order to adjust the transmitted light passing through the HPDLCs. Main advantages of such systems are low cost, high resolution, high contrast, full color and fast switch-on/switch-off durations. However major disadvantage of HPDLCs is high control voltage and limited viewing angle. This technology will be one of the most promising technologies for holographic displays if the level of control voltages is decreased. Another promising technology, which is not common, is AOMs. AOMs are widely used for applications other than holographic displays and therefore fundamentals of the underlying technology is well known. Time-varying holographic patterns can be generated by using SAWs. This is one of the research fields for future holographic display systems.

The first electro-holographic video display system, called Mark-I, was developed by the Spatial Imaging Group at the MIT Media Lab in 1989, and it has been improving over the past 20 years by incorporating multi-AOM or multi-SAW devices. This approach, which uses such acoustic wave devices, has strong advantages such as a wide diffraction angle, high diffraction efficiency, and high available spatial frequency. However, these devices are rarely used since they modulate the light only in one dimension. LC SLMs are more suitable for electro-holographic display systems because they are two dimensional modulators and they are easier to use. Therefore, even if the diffraction angle is not sufficient, many holographic display prototypes use various LC SLMs. SeeReal technologies has developed a prototype with a 20 inch commercial LCD monitor. The distance between the pixel centers in this prototype is about $200 \mu \mathrm{m}$, and the diffraction angle is less than 0.2 degrees. They chose a large reconstruction volume of the 3D scene instead of a wide viewing zone. The depth perception that the system provides is impressive. Although the viewing window for each eye is less than $10 \mathrm{~mm} \times 10 \mathrm{~mm}$, an eye-tracking system provides the necessary flexibility. The observer window is shifted to the actual eye position by movement of the light source. However this method is only for a single observer. A future holographic display system must accommodate not only a large reconstruction volume but also a wide viewing window in order to replace conventional $2 \mathrm{D}$ display systems. A prototype called "active tiling system" is developed by QinetiQ. This system exploits high frame rate EASLMs and high resolution OASLMs. They use spatial-multiplexing method to obtain $3 \times 8$ billion-pixel holographic display and they achieved full-parallax and full-color reconstructions. On the other hand, a huge computational resource is required to generate a huge size holographic fringe pattern in real time. They used more than 100 computers for this prototype. There are several ways to solve this computational problem. A typical dedicated CGH calculator is called Horn which uses an FPGA. The HORN-6, latest version, has four FPGA chips and 256 megabyte DDR-SDRAM for calculation. Using the HORN-6 cluster system composed of 16 HORN-6 boards, researchers have generated two megapixel frame size digital holograms at a rate of one frame per second for objects with one million points. The approach using the FPGA technology can provide an excellent computational performance. However, this approach has problems associated with the high cost, long development time, and complicated programming.

A typical approximation method for fast digital hologram generation is the coherent holographic stereogram. Computational speed of the coherent holographic stereogram is much faster than that of the reconstructions from direct methods. In addition, the latest version called ACPAS has higher quality reconstructed image, and generates more accurate results. Since the algorithm of the coherent stereogram can be easily mapped to a single instruction multiple data architecture, the ACPAS computing can significantly benefit from GPU based implementations. By using this method, one megapixel frame size full color holograms can be generated as a rate of $30 \mathrm{fps}$ for objects with more than 10000 points. However, only point cloud corresponding to a $3 \mathrm{D}$ computer graphic model is allowed as the input information. A different hologram type for holographic video is the image hologram. The characteristic of this hologram is to place an image of the 3D object close to hologram plane. Therefore, the computational complexity for digital hologram generation can be reduced. But this technique can be used for only thin objects because the depth range is limited.

Electro-holography is one of the advanced techniques in 3D display technologies. This survey paper presents the current research activities on holographic displays together with related commercial systems.

\section{REFERENCES}

[1] D. Gabor, "A new microscopic principle," Nature, vol. 161, pp. 777-778, 1948.

[2] D. Gabor, "Microscopy by reconstructed wavefronts," Proc. Roy. Soc. A, vol. 197, pp. 454-487, 1949.

[3] D. Gabor, "Microscopy by reconstructed wavefronts: II," Proc. Roy. Soc. B, vol. 64, pp. 449-469, 1951.

[4] E. N. Leith and J. Upatnieks, "Reconstructed wavefronts and communication theory," J. Opt. Soc. Am., vol. 52, pp. 1123-1130, 1962.

[5] E. N. Leith and J. Upatnieks, "Wavefront reconstruction with continuous-tone objects," J. Opt. Soc. Amer., vol. 53, pp. 1377-1381, 1963.

[6] E. N. Leith and J. Upatnieks, "Wavefront reconstruction with diffused illumination and three-dimensional objects," J. Opt. Soc. Amer., vol. 54, pp. 1295-1301, 1964. 
[7] Y. N. Denisyuk, "Photographic reconstruction of the optical properties of an object in its own scattered field," Sov. Phys. Dokl., vol. 7, p. 543, 1962.

[8] T. Kreis, Handbook of Holographic Interferometry: Optical and Digital Methods. Hoboken, NJ: Wiley, 2005.

[9] A. W. Lohmann and D. Paris, "Binary Fraunhofer holograms generated by computer," Appl. Opt., vol. 6, pp. 1739-1748, 1967.

[10] J. W. Goodman and R. W. Lawrence, "Digital image formation from electronically detected holograms," Appl. Phys. Lett., vol. 11, pp. 77-79, 1967.

[11] L. Onural, A. Gotchev, H. Ozaktas, and E. Stoykova, "A survey of signal processing problems and tools in holographic three-dimensional television," IEEE Trans. Circuits Syst. Video Technol., vol. 17, p. 1631, 2007, IEEE.

[12] P. Benzie, J. Watson, P. Surman, I. Rakkolainen, K. Hopf, H. Urey, V. Sainov, and C. von Kopylow, "A survey of 3DTV displays: Techniques and technologies," IEEE Trans. Circuits Syst. Video Technol., vol. 17, no. , p. 1647, 2007.

[13] S. A. Benton, "Survey of holographic stereograms," Proc. SPIE, vol. 367, pp. 15-19, 1983.

[14] A. J. van Dekker and A. van den Bos, "Resolution: A survey," J. Opt., vol. 14 , pp. 547-557, 1997.

[15] S. Barnard and M. Fischler, "Computational stereo," ACM Comput. Surveys (CSUR), vol. 14, pp. 553-572, 1982.

[16] C. Zhang and T. Chen, "A survey on image-based rendering-Representation, sampling and compression," Signal Process: Image Commun., vol. 19, pp. 1-28, 2004.

[17] P. L. Butzer, "A survey of Whittaker-Shannon sampling theorem and some of its extensions," J. Math. Res. Expo., vol. 3, pp. 185-212, 1983.

[18] K. Bauchert, S. Serati, and A. Furman, "Advances in liquid crystal spatial light modulators," Proc. SPIE, vol. 4734, pp. 35-43, 2002.

[19] A. Michalkiewicz, M. Kujawinskaa, T. Kozackia, X. Wangb, and P. J. Bosb, "Holographic three-dimensional displays with liquid crystal on silicon spatial light modulator," Proc. SPIE, vol. 5531, pp. 85-94, 2004.

[20] Y. Frauel, T. J. Naughton, O. Matoba, E. Tajahueerce, and B. Javidi, "Three-dimensional imaging and processing using computational holographic imaging," in Proc. IEEE, 2006, vol. 94, p. 636, IEEE.

[21] T. Ito, T. Shimobaba, H. Godo, and M. Horiuchi, "Holographic reconstruction with a $10-\mu \mathrm{m}$ pixel-pitch reflective liquid-crystal display by use of a light-emitting diode reference light," Opt. Lett., vol. 27, pp. 1406-1408, 2002.

[22] T. Shimobaba and T. Ito, "A color holographic reconstruction system by time division multiplexing with reference lights of laser," Opt. Rev., vol. 10 , no. 5, pp. 339-341, 2003.

[23] T. Shimobaba, A. Shiraki, N. Masuda, and T. Ito, "An electroholographic colour reconstruction by time division switching of reference lights," J. Opt. A: Pure Appl. Opt., vol. 9, pp. 757-760, 2007.

[24] K. Sato, A. Sugita, M. Morimoto, and K. Fujii, "Reconstruction of fullcolor images with a RGB time-sharing holographic display system," in Proc. SPIE, 2006, vol. 6030, p. 603004.

[25] K. Sato, A. Sugita, M. Morimoto, and K. Fujii, "Reconstruction of color images of high quality by a holographic display," in Proc. SPIE, 2006, vol. 6136, p. $61360 \mathrm{~V}$.

[26] A. Sugita, K. Sato, M. Morimoto, and K. Fujii, "Full-color holographic display and recording of 3D images," in Proc. SPIE, 2005, vol. 5742, p. 130.

[27] T. Ito and K. Okano, "Color electroholography by three colored reference lights simultaneously incident upon one hologram panel," Opt. Expr., vol. 12, pp. 4320-4325, 2004.

[28] N. Ohmura, H. Kang, T. Yamaguchi, and H. Yoshikawa, "A method to increase the hologram viewing angle by the beam reconfiguration," in Proc. SPIE, 2008, vol. 6912, p. 69120 O.

[29] H. Kang, N. Ohmura, T. Yamaguchi, H. Yoshikawa, S.-C. Kim, and E.-S. Kim, "Method to enlarge the hologram viewing window using a mirror module," Opt. Eng., vol. 48, no. 7, p. 075801, 2009.

[30] K. Takano, N. Minami, and K. Sato, "A simple method of color electroholographic display system using a white light source and three LCD panels," in IEEE Int. Conf. on Multimedia and Expo, 2001, p. 105, IEEE.

[31] K. Takano and K. Sato, "Color electro-holographic display using a single white light source and a focal adjustment method," Opt. Eng., vol. 41 , pp. 2427-2433, 2002.

[32] K. Takano, N. Minami, and K. Sato, "Practical method for color electro-holographic display system using a metal halide lamp and three LCD panels," in Proc. SPIE, 2002, vol. 4659, p. 61.
[33] K. Sato and K. Takano, "New type electro-holographic display system using LCDs," in Proc. SPIE, 2002, vol. 4864, p. 114.

[34] K. Takano and K. Sato, "Full-color electroholographic three-dimensional display system employing light emitting diodes in virtual image reconstruction," Opt. Eng., vol. 46, p. 095801, 2007.

[35] K. Sato and K. Takano, "Consideration about HMD-type holography 3D-TV," in Proc. SPIE, 2004, vol. 5599, p. 123

[36] T. Yamaguchi and H. Yoshikawa, "Real time calculation for holographic video display," in Proc. SPIE, 2006, vol. 6136, p. 61360T.

[37] T. Yamaguchi, G. Okabe, and H. Yoshikawa, "Full-color image-plane holographic video display," in Proc. SPIE, 2007, vol. 6488, p. 64880Q.

[38] S. Kim, J. Moon, E. K. D. W. Lee, and K. C. Son, "Holographic fullcolor 3D display system using color-LCoS spatial light modulator," in Proc. SPIE, 2005, vol. 5742, p. 223.

[39] J. Moon, D. Lee, S. Kim, and E. Kim, "Color LCoS-based full-color electro-holographic 3D display system," in Proc. SPIE, 2005, vol. 5801, p. 294.

[40] F. Yaras, M. Kovachev, R. Ilieva, M. Agour, and L. Onural, "Holographic reconstructions using phase-only spatial light modulators," in 3DTV Conf.: The True Vision-Capture, Transmission and Display of $3 D$ Video, 2008, p. PD-1, IEEE.

[41] F. Yaras and L. Onural, "Color holographic reconstruction using multiple SLMs and LED illumination," in Proc. SPIE, 2009, vol. 7237, p. 723700.

[42] R. Tudela, I. Labastida, E. Marti-Badosa, S. Vallmitjana, I. Juvells, and A. Carnicer, "A simple method for displaying Fresnel holograms on liquid crystal panels," Opt. Commun., vol. 214, pp. 107-114, 2002.

[43] R. Tudela, E. Martin-Badosa, I. Labastida, S. Vallmitjana, I. Juvells, and A. Carnicer, "Full complex Fresnel holograms displayed on liquid crystal devices," J. Opt. A: Pure Appl. Opt., vol. 5, pp. 189-194, 2003.

[44] R. Tudela, E. Martin-Badosa, I. Labastida, S. Vallmitjana, and A. Carnicer, "Wavefront reconstruction by adding modulation capabilities of two liquid crystal devices," Opt. Eng., vol. 43, no. 11, pp. 2650-2657, 2004.

[45] P. V. Yezhov and A. V. Kuzmenko, "Synthesized phase objects instead of real ones for optical-digital recognition systems," in Proc. SPIE, 2004, vol. 5477, p. 412.

[46] P. V. Yezhov, A. V. Kuzmenko, T. N. Smirnova, and A. A. Ivanovskyy, "Synthesized phase objects used instead of real ones for optical-digital recognition systems: Experiment," in Proc. SPIE, 2006, vol. 6254, p. 625419.

[47] A. V. Kuzmenko and P. V. Yezhov, "Iterative algorithms for off-axis double-phase computer-generated holograms implemented with phase-only spatial light modulators," Appl. Opt., vol. 46, pp. 7392-7400, 2007.

[48] L. B. Lesem, P. M. Hirsch, and J. A. J. Jr, "The kinoform: A new wavefront reconstruction device," IBM J. Res.Develop., vol. 13, pp. 150-155, 1969.

[49] N. Yoshikawa and T. Yatagai, "Phase optimization of a kinoform by simulated annealing," Appl. Opt., vol. 33, pp. 863-868, 1994.

[50] J. Amako and T. Sonehara, "Kinoform using an electrically controlled birefringent liquid-crystal spatial light modulator," Appl. Opt., vol. 30, p. 4622, 1991.

[51] A. V. Kuzmenko and P. V. Yezhov, "Iterative Fourier-transform algorithm of synthesis of a kinoform with the use of the operation of predistortion of an object," in Proc. SPIE, 2008, vol. 7008, p. 70081W.

[52] A. V. Kuzmenko, "Weighting iterative Fourier transform algorithm of the kinoform synthesis," Opt. Lett., vol. 33, pp. 1147-1149, 2008.

[53] M. Makowski, M. Sypek, A. Kolodziejczyk, G. M. a, and J. aw Suszek, "Iterative design of multiplane holograms: Experiments and applications," Opt. Eng., vol. 46, p. 045802, 2007.

[54] T. Nakamura, K. Nitta, and O. Matoba, "Iterative algorithm of phase determination in digital holography for real-time recording of real objects," Appl. Opt., vol. 46, pp. 6849-6853, 2007.

[55] D. Palima and V. Daria, "Holographic projection of arbitrary light patterns with a suppressed zero-order beam," Appl. Opt., vol. 46, pp. 4197-4201, 2007.

[56] K. Choi, J. Kim, Y. Lim, and B. Lee, "Full parallax viewing-angle enhanced computer-generated holographic 3D display system using integral lens array," Opt. Expr., vol. 13, pp. 10494-10502, 2005.

[57] J. Hahn, H. Kim, Y. Lim, G. Park, and B. Lee, "Wide viewing angle dynamic holographic stereogram with a curved array of spatial light modulators," Opt. Expr., vol. 16, pp. 12372-12386, 2008.

[58] A. Jesacher, C. Maurer, A. Schwaighofer, S. Bernet, and M. RitschMarte, "Near-perfect hologram reconstruction with a spatial light modulator," Opt. Expr., vol. 16, pp. 2597-2603, 2008. 
[59] T. Kreis, P. Aswendt, and R. Hofling, "Hologram reconstruction using a digital micromirror device," Opt. Eng., vol. 40, pp. 926-933, 2001.

[60] M. Kovachev, R. Ilieva, L. Onural, G. B. Esmer, T. Reyhan, P. Benzie, J. Watson, and E. Mitev, "Reconstruction of computer generated holograms by spatial light modulators," in MRCS, 2006, pp. 706-713.

[61] M. Kovachev, R. Ilieva, P. Benzie, G. B. Esmer, L. Onural, J. Watson, and T. Reyhan, "Holographic 3DTV displays using spatial light modulators," in Three-Dimensional Television-Capture, Transmission, Display, H. Ozaktas and L. Onural, Eds. : Springer, 2008, pp. 529-555.

[62] P. Birch, R. Young, C. Chatwin, M. Farsari, D. Budgett, and J. Richardson, "Fully complex optical modulation with an analogue ferroelectric liquid crystal spatial light modulator," Opt. Commun., vol. 175 , pp. 347-352, 2000.

[63] C. Stolz, L. Bigue, and P. Ambs, "Implementation of high-resolution diffraction optical elements on coupled phase and amplitude spatial light modulators," Appl. Opt., vol. 40, no. 35, pp. 6415-6424, 2001.

[64] P. Birch, R. Young, D. Budgett, and C. Chatwin, "Dynamic complex wave-front modulation with an analog spatial light modulator," Opt. Lett., vol. 26, no. 12, pp. 920-922, 2001.

[65] P. Grother and D. Casasent, "Optical path diffrence measurement techniques for SLMs," Opt. Commun., vol. 189, pp. 31-38, 2001.

[66] M. Yamauchi, "Origin and characteristics of ambiguous properties in measuring physical parameters of twisted nematic liquid crystal spatial light modulators," Opt. Eng., vol. 41, no. 5, pp. 1134-1141, 2002.

[67] J. Nicolas and J. A. Davis, "Programmable wave plates using a twisted nematic liquid crystal display," Opt. Eng., vol. 41, no. 12, pp. 3004-3005, 2002.

[68] C. Kohler, X. Schwab, and W. Osten, "Optimally tuned spatial light modulators for digital holography," Appl. Opt., vol. 45, pp. 960-967, 2006.

[69] A. Jesacher, A. Schwaighofer, S. Furhapter, C. Maurer, S. Bernet, and M. Ritsch-Marte, "Wavefront correction of spatial light modulators using an optical vortex image," Opt. Express, vol. 15, pp. 5801-5808, 2007.

[70] E. G. van Putten, I. M. Vellekoop, and A. P. Mosk, "Spatial amplitude and phase modulation using commercial twisted nematic LCDs," Appl. Opt., vol. 47, pp. 2076-2081, 2008.

[71] J. Otón, P. Ambs, M. S. Millán, and E. Pérez-Cabré, "Multipoint phase calibration for improved compensation of inherent wavefront distortion in parallel aligned liquid crystal on silicon displays," Appl. Opt., vol. 46, pp. 5667-5679, 2007.

[72] F. Reinitzer, "Beitrge zur kenntniss des cholesterins," Wiener Monatschr, Fur Chem., vol. 9, pp. 421-441, 1888.

[73] J. Neff, R. Athale, and S. Lee, "Two-dimensional spatial light modulators: A tutorial," Proc. IEEE, vol. 78, no. , pp. 826-855, 1990.

[74] S. T. Tang, F. H. Yu, J. Chen, M. Wong, H. C. Huang, and H. S. Kwok, "Reflective twisted nematic liquid crystal displays. I. Retardation compensation," J. Appl. Phys., vol. 81, pp. 5924-5929, 1997.

[75] H. Dai, K. X. Y. Liu, X. Wang, and J. Liu, "Characteristics of LCoS phase-only spatial light modulator and its applications," Opt. Commun., vol. 238, pp. 269-276, 2004.

[76] S. Osten, S. Krger, and A. Steinhoff, "Spatial light modulators based on reflective micro-displays," Techn. Messen, vol. 73, pp. 149-156, 2006.

[77] O. Trushkevych, N. Collings, W. A. Crossland, and T. D. Wilkinson, "Resolution in optically addressed spatial light modulators based on dye-doped liquid crystals," Appl. Opt., vol. 45, pp. 8889-8892, 2006.

[78] J. B. Sampsell, "Digital micromirror device and its application to projection displays," J. Vac. Sci. Technol. B, vol. 12, pp. 3242-3246, 1994.

[79] R. T. Pogue, R. L. Sutherland, M. G. Schmitt, L. V. Natarajan, S. A. Siwecki, V. P. Tondiglia, and T. J. Bunning, "Electrically switchable Bragg gratings from liquid crystal/polymer composites," Appl. Spectrosc., vol. 54, pp. 12A-28A, 2000.

[80] J.-H. Park, H.-R. Kim, Y. Kim, J. Kim, J. Hong, S.-D. Lee, and B. Lee, "Depth-enhanced three-dimensional-Two-dimensional convertible display based on modified integral imaging," Opt. Lett., vol. 29, pp. 2734-2736, 2004.

[81] Y. Sarov, T. Angelov, and S. Sainov, "Optical properties of polymer dispersed cholesteric liquid crystals," Bulg. J. Phys., vol. 31, pp. 33-38, 2004.

[82] K. Beev, S. Sainov, T. Angelov, and A. G. Petrov, "Investigation of Bragg gratings recorded in polymer-dispersed liquid crystals," J. Optoelectron. Adv. Mater., vol. 6, pp. 799-803, 2004.

[83] K. Beev, L. Criante, D. E. Lucchetta, F. Simoni, and S. Sainov, "New holographic gratings recorded in polymer-dispersed liquid crystals," in Proc. SPIE, 2006, vol. 6252, p. 62522B.
[84] J. S. Kollin, S. A. Benton, and M. L. Jepsen, "Real-time display of 3-D computed holograms by scanning the image of an acousto-optic modulator," in Proc. SPIE, 1989, vol. 1136, p. 178.

[85] P. S.-Hilaire, S. A. Benton, M. Lucente, and P. M. Hubel, "Color images with the MIT holographic video display," in Proc. SPIE, 1992, vol. 1667 , p. 73.

[86] L. Onural, G. Bozdağı, and A. Atalar, "New high-resolution display device for holographic three-dimensional video: Principles and simulations," Opt. Eng., vol. 33, pp. 835-844, 1994.

[87] P. St.-Hilaire, "Scalable Optical Architectures for Electronic Holography," Ph.D. dissertation, Program in Media Arts and Sciences, MIT, Cambridge, 1994.

[88] P. St-Hilaire, S. A. Benton, M. Lucente, J. D. Sutter, and W. J. Plesniak, "Advances in holographic video," in Proc. SPIE, 1993, vol. 1914, p. 188.

[89] P. St.-Hillaire, M. Lucente, J. D. Sutter, R. Pappu, C. J. Sparrell, and S. Benton, "Scaling up the MIT holographic video system," in Proc. SPIE, 1995, vol. 2333, p. 374

[90] M. L. Jepsen, "Holographic video: design and implementation of a display system," M.S. thesis, Dep. Architecture, MIT, Cambridge, 1989.

[91] J. S. Kollin, "Design and information considerations for holographic television," M.S. Thesis, Dep. Architecture, MIT, Cambridge, 1988.

[92] M. Lucente and T. Galyean, "Rendering interactive holographic images," in Proc. SIGGRAPH'95, 1995, pp. 387-394.

[93] J. Underkoffler, "Towards accurate computation of optically reconstructed holograms," M.S. thesis, Program in Media Arts and Sciences, MIT, Cambridge, 1991

[94] D. E. Smalley, Q. Y. J. Smithwick, and J. V. Michael Bove, "Holographic video display based on guided-wave acousto-optic devices," in Proc. SPIE, 2007, vol. 6488, p. 64880L.

[95] S. Reichelt, R. Haussler, N. Leister, G. Futterer, and A. Schwerdtner, "Large holographic 3D displays for tomorrow's TV and monitors-Solutions, challenges, and prospects," in IEEE 2008. LEOS 21st Annu. Meeting, 2008, p. 194, IEEE.

[96] R. Häussler, A. Schwerdtner, and N. Leister, "Large holographic displays as an alternative to stereoscopic displays," in Proc. SPIE, 2008, vol. 6803 , p. 68030M.

[97] S. Reichelt, H. Sahm, N. Leister, and A. Schwerdtner, "Capabilities of diffractive optical elements for real-time holographic displays," in Proc. SPIE, 2008, vol. 6912, p. 69120P.

[98] A. Schwerdtner, R. Häussler, and N. Leister, "Large holographic displays for real-time applications," in Proc. SPIE, 2008, vol. 6912, p. 69120T.

[99] N. Leister, A. Schwerdtner, G. Fütterer, S. Buschbeck, J.-C. Olaya, and S. Flon, "Full-color interactive holographic projection system for large 3D scene reconstruction," in Proc. SPIE, 2008, vol. 6911, p. 69110V.

[100] M. Stanley, M. A. Smith, A. P. Smith, P. J. Watson, S. D. Coomber, C. D. Cameron, C. W. Slinger, and A. D. Wood, "3D electronic holography display system using a 100 mega-pixel spatial light modulator,' in Proc. SPIE, 2004, vol. 5249, p. 297.

[101] M. Stanley, P. Conway, S. Coomber, J. Jones, D. Scattergood, C. Slinger, B. Bannister, C. Brown, W. Crossland, and A. Travis, "A novel electro-optic modulator system for the production of dynamic images from giga-pixel computer generated holograms," in Proc. SPIE, 2000, vol. 3956, p. 13

[102] C. Slinger, C. Cameron, and M. Stanley, "Computer-generated holography as a generic display technology," Computer, vol. 38 , no. 8 , pp. 46-53, 2005, IEEE.

[103] T. Shimobaba, S. Hishinuma, and T. Ito, "Special-purpose computer for holography HORN-4 with recurrence algorithm," Comput. Phys. Commun., vol. 148, pp. 160-170, 2002.

[104] T. Yabe, T. Ito, and M. Okazaki, "Holography machine HORN-1 for computer-aided retrieval of virtual three-dimensional image," Jpn. $J$. Appl. Phys. Lett., vol. 32, pt. 2, pp. L1359-L1361, 1993.

[105] Y. Ichihashi, H. Nakayama, T. Ito, N. Masuda, T. Shimobaba, A Shiraki, and T. Sugie, "HORN-6 special-purpose clustered computing system for electroholography," Opt. Express, vol. 17, pp. 13895-13903, 2009.

[106] T. Ito, N. Masuda, K. Yoshimura, A. Shiraki, T. Shimobaba, and T. Sugie, "Special-purpose computer HORN-5 for a real-time electroholography," Opt. Express, vol. 13, pp. 1923-1932, 2005.

[107] T. Shimobaba, N. Masuda, T. Sugie, S. Hosono, S. Tsukui, and T. Ito, "Special-purpose computer for holography HORN-3 with PLD technology," Comput. Phys. Commun., vol. 130, July 2000. 
[108] T. Ito, T. Yabe, M. Okazaki, and M. Yanagi, "Special-purpose computer HORN-1 for reconstruction of virtual image in three dimensions," Comput. Phys. Commun., vol. 82, pp. 104-110, 1994.

[109] T. Ito, H. Eldeib, K. Yoshida, S. Takahashi, T. Yabe, and T. Kunugi, "Special-purpose computer for holography HORN-2," Comput. Phys. Commun., vol. 93, pp. 13-20, 1996.

[110] T. Hamano and H. Yoshikawa, "Image-type CGH by means of e-beam printing," in Proc. SPIE, 1998, vol. 3293, p. 2.

[111] T. Yamaguchi, G. Okabe, and H. Yoshikawa, "Real-time image plane full-color and full-parallax holographic video display system," Opt. Eng., vol. 46, p. 125801, 2007.

[112] T. Yamaguchi and H. Yoshikawa, "Real time calculation for holographic video display," in Proc. SPIE, 2006, vol. 6136, p. 61360T.

[113] H. Yoshikawa, T. Yamaguchi, and R. Kitayama, "Real-time generation of full color image hologram with compact distance look-up table," in OSA Tech. Dig. Digital Holography and Three-Dimensional Imaging, 2009, paper DWC4.

[114] T. Yatagai, "Stereoscopic approach to 3-D display using computer-generated holograms," Appl. Opt., vol. 15, pp. 2722-2729, 1976.

[115] H. Kang, T. Yamaguchi, H. Yoshikawa, S.-C. Kim, and E.-S. Kim, "Acceleration method of computing a compensated phase-added stereogram on a graphic processing unit," Appl. Opt., vol. 47, pp. 5784-5789, 2008.

[116] H. Kang, T. Fujii, T. Yamaguchi, and H. Yoshikawa, "Compensated phase-added stereogram for real-time holographic display," Opt. Eng., vol. 46, p. 095802, 2007.

[117] H. Y. J. Tamai, "Faster computation of subsampled coherent stereogram (in japanese)," J. Inst. Television Engineers Jpn., vol. 50, pp. $1612-1615,1996$.

[118] M. Yamaguchi, H. Hoshino, T. Honda, and N. Ohyama, "Phase-added stereogram: Calculation of hologram using computer graphics technique," in Proc. SPIE, 1993, vol. 1914, p. 25.

[119] H. Yoshikawa and H. Kameyama, "Integral holography," in Proc. SPIE, 1995, vol. 2406, p. 226.

[120] H. Kang, "Quality Improvements of the coherent holographic stereogram for natural 3D display and its applications," $\mathrm{Ph} . \mathrm{D}$. dissertation, Nihon University, Tokyo, Japan, 2008.

[121] H. Kang, F. Yaraş, and L. Onural, "Graphics processing unit accelerated computation of digital holograms," Appl. Opt., vol. 48, no. 34, pp. H137-H143, 2009.

[122] F. Yaraş, H. Kang, and L. Onural, "Real-time phase-only color holographic video display system using LED illumination," Appl. Opt., vol. 48, no. 34, pp. H48-H53, 2009.

[123] H. Kang, F. Yaraş, L. Onural, and H. Yoshikawa, "Real-time fringe pattern generation with high quality," in OSA Tech. Dig. Digital Holography and Three-Dimensional Imaging, 2009, paper DTuB7.

[124] F. Yaraş, H. Kang, and L. Onural, "Real-time multiple SLM color holographic display using multiple GPU acceleration," in OSA Tech. Dig. Digital Holography and Three-Dimensional Imaging, 2009, paper DWA4.

[125] H. Kang, F. Yaras, and L. Onural, "Quality comparison and acceleration for digital hologram generation method based on segmentation," in Proc. IEEE 3DTV Conf.: The True Vision-Capture, Transmission and Display of $3 D$ Video, 2009.

[126] F. Yaras, H. Kang, and L. Onural, "Real-time color holographic video display system," in Proc. IEEE 3DTV Conf.: The True Vision-Capture, Transmission and Display of 3D Video, 2009.

[127] M. Schubin, NAB 2009: Holography Update [Online]. Available: http://www.televisionbroadcast.com/article/79134
[128] J.-C. Grossetie, "Computer-assisted method and device for restoring three-dimensional images," U.S. Patent 6621 605, 2003.

[129] T. Balogh, Z. Dobranyi, T. Forgacs, A. Molnar, L. Szloboda, E. Gobbetti, F. Marton, F. Bettio, G. Pintore, G. Zanetti, E. Bouvier, and R. Klein, "An interactive multi-user holographic environment," in $A C M$ SIGGRAPH'06 Emerging Technologies, 2006, p. 18.

[130] Z. Megyesi, A. Barsi, and T. Balogh, "3D video visualization on the holovizio system," in Proc. IEEE 3DTV Conf.: The True Vision-Capture, Transmission and Display of 3D Video, 2008, p. 269.

[131] T. Balogh, P. Kovacs, and A. Barsi, "Holovizio 3D display system," in Proc. IEEE 3DTV Conf.: The True Vision-Capture, Transmission and Display of 3D Video, 2007.

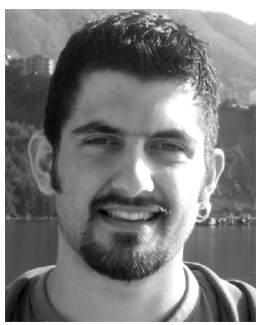

Fahri Yaras (GSM'10) received the B.S. degree in electrical and electronics engineering from Bilkent University, Ankara, Turkey, in 2005, where he is currently working toward the Ph.D. degree.

He was a member of EC funded 3DTV Project (2005-2008). Now he is a member of EC funded Real 3D Project. His research interests are 3DTV, holographic 3DTV, 3D displays, electro-holography and optical signal processing. He is a member of IEEE and OSA.

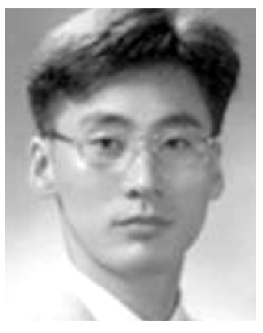

Hoonjong Kang received the B.Eng. and M.Eng. degrees in electrical engineering from Kwangwoon University, Seoul, Korea, in 1998, and 2001, respectively, and the Ph.D. degree in engineering degree from Nihon University, Tokyo, Japan, in 2008

He joined the 3D Korea company in 2000, and joined the government institute ETRI in 2002. He joined the European FP7 Real3D project in 2008. His research interests include computer-generated holograms, electro-holography, 3-D display, and signal processing of stereoscopic images.

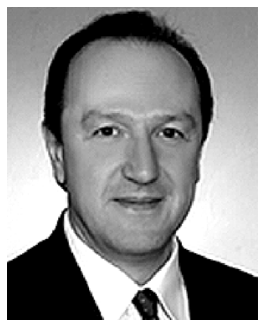

Levent Onural (S'82-M'85-SM'91-F'08) received the B.S. and M.S. degrees in electrical engineering from METU in 1979 and 1981, respectively, and the $\mathrm{Ph} . \mathrm{D}$. degree in electrical and computer engineering from SUNYAB in 1985.

He joined the EEE Department of Bilkent, Ankara, Turkey, in 1987 where he is currently a Full Professor. His current research interests are in image and video processing, with emphasis on video coding, 3DTV, holographic 3DTV and signal processing aspects of optical wave propagation. He was the coordinator of EC funded 3DTV Project (2004-2008). He is the co-leader of 3D Media Cluster which is an umbrella organization formed by many European Commission funded 3D-related projects.

Dr. Onural received a Third Millenium Medal from IEEE in 2000. He served IEEE as Director of IEEE Region 8 (2001-2002), Secretary of IEEE (2003), and a member of IEEE BoD (2001-2003). He was a Fulbright scholar (1981-1985). 